TOPICAL REVIEW

\title{
Astrophysical evidence for the existence of black holes
}

\author{
A Celotti, J C Miller and D W Sciama \\ SISSA, Via Beirut 2-4, 34013 Trieste, Italy.
}

\begin{abstract}
Following a short account of the history of the idea of black holes, we present a review of the current status of the search for observational evidence of their existence aimed at an audience of relativists rather than astronomers or astrophysicists. We focus on two different regimes: that of stellar-mass black holes and that of black holes with the masses of galactic nuclei.
\end{abstract}

Date: 1 February 2008 


\section{Introduction}

In his famous article of 1784 , which is seen as being the beginning of the story of black holes, John Michell [1] wrote:

If there should really exist in nature any [such] bodies, ... we could have no information from sight; yet, if any other luminous bodies should happen to revolve about them we might still perhaps from the motions of these revolving bodies infer the existence of the central ones with some degree of probability, as this might afford a clue to some of the apparent irregularities of the revolving bodies, which would not be easily explicable on any other hypothesis.

There at the very beginning, the theoretically-predicted properties of (Newtonian) black holes were discussed together with a carefully-worded statement about how it might be determined observationally whether such objects do in fact exist. Following Michell's paper and the subsequent repetition of his arguments by Laplace [2] in his book of 1796, there is a long gap until the present century when, with the coming of general relativity, the theoretical discussion of black holes started anew. The observational search had to wait rather longer, until the development of radio astronomy (from the late 1940s onwards) and X-ray astronomy (from the 1960s onwards).

The basic theory of black holes is now well-understood, but the path to this understanding has been long and tortuous. It has been beautifully described by Israel [3] in the book "300 Years of Gravitation". The first black-hole solution of Einstein's field equations was discovered in heroic circumstances by Karl Schwarzschild [4] who was looking for the exact solution for a point mass in otherwise empty space. He discovered his solution only two months after the publication in 1915 of Einstein's definitive paper on general relativity [5], while serving in the German army. He died shortly thereafter.

The black hole property of the Schwarzschild solution was soon recognised; it was certainly known to Sir Oliver Lodge in 1921 [6]. However, progress in understanding (and accepting) black holes was slow - people were unwilling to accept that actual physical objects would ever collapse to such an extreme state. Another stumbling block rested on a mathematical misunderstanding springing from the obvious fact that in the usually adopted Schwarzschild co-ordinates the metric becomes singular at the event horizon $r=r_{s}=2 G M / c^{2}$ (where $M$ is the mass of the black hole). Also, a further cause for the delay in understanding came from the fact that in the thirties people did not seem to pay much attention to the discoveries reported by other people.

One discovery which was attended to, but was found to be rather alarming, was Chandrasekhar's crucial result of 1931 [7] that relativistic degeneracy of electrons could not prevent a cold body more massive than $\sim 1.44 M_{\odot}$ (where $M_{\odot}$ denotes the mass of the sun) from collapsing indefinitely. Much later, after the alarm was 
dissipated, Chandrasekhar was awarded a share in the Nobel prize for this discovery. An influential figure whose alarm at the time was acute was Sir Arthur Eddington, who famously said in 1935 [8] after referring to Chandrasekhar's result: "Various accidents may intervene to save the star, but I want more protection than that. I think there should be a law of Nature to prevent a star from behaving in this absurd way!".

In 1933 Lemaitre [9] had already noted that the Schwarzschild singularity was a mathematical artefact on the same footing as certain singularities in cosmological metrics which puzzled people for many years. Yet we find six years later no less a person than Einstein himself [10] writing a fifteen page paper, full of calculations arguing that a physical system would necessarily have to be larger than the radius at which the "undesirable" Schwarzschild singularity would otherwise occur.

In that same year (1939) Oppenheimer and Snyder [11] wrote a definitive paper in a prominent journal (Physical Review) showing that a spherically symmetric pressurefree mass would collapse indefinitely, and correctly described the effect on light propagation in the neighbourhood of the event horizon. Yet the authoritative textbook on general relativity by Bergmann [12] published three years later does not mention the crucial Oppenheimer-Snyder result, but does spend a whole page on Einstein's paper.

The modern epoch of understanding commenced in the nineteen fifties, partly from the introduction of coordinate systems in which the metric is regular everywhere except at the origin (of which the Kruskal metric [13] is the best known) and partly from John Wheeler's relentless emphasis on the importance of understanding the final stages of gravitational collapse [14]. Other highlights were Kerr's discovery [15] of an exact solution for rotating black holes, and Israel's uniqueness theorem [16] for nonrotating black holes, soon to be extended by Carter and others [17] to the rotating case. Another spectacular result was Penrose's 1965 singularity theorem [18] which showed that formation of physical singularities (such as that at $r=0$ for Schwarzschild black holes) was a generic feature of continued collapse, contradicting the widely held view that the occurrence of a singularity was a special feature related to exact spherical symmetry. Penrose's result was soon extended to cosmological space-times by Hawking [19] and by Geroch [20].

With hindsight we can see that, once it was understood what is the appropriate level of mathematical sophistication to adopt for research on black holes, the stage was set for the great complex of discoveries that were made in the nineteen fifties and sixties, mainly by the considerable number of highly talented young mathematicians and physicists who were attracted to enter the field in those years.

Nevertheless there was still a shock to come. The widening of people's mental horizons did not prepare them for perhaps the greatest discovery yet made about black holes, namely the Hawking radiation [21] which they are predicted to emit when quantum effects are taken into account and which permits a black hole thermodynamics to be set up. This wonderful result, published in 1974, was received with widespread incredulity, but only for a short time. Perhaps people were learning 
the important lesson that they should consider what a well-established theory says in an open-minded way and not be blinded by possibly mistaken personal intuition.

So, where do we stand on the question of whether black holes as described by the mathematical solutions discussed above actually exist in nature? For the remainder of this article, we will be concentrating on this question.

In the second half of the twentieth century, relativistic astrophysics in general and the study of black holes in particular has changed from being a fairly marginal minority interest as far as astronomers were concerned, to being in the central mainstream of astrophysical research. This change has been closely connected with advances in technology: the advent of radio and X-ray astronomy (as already noted) and also,later, the rise of numerical computing which has allowed relativistic calculations to be made for more complex physical situations than would be possible analytically, permitting closer confrontation with observations.

What are the circumstances in which astrophysical black holes are thought likely to be formed? The "mean density" $\bar{\rho}$ of a black hole (its mass $M$ divided by $\frac{4}{3} \pi r_{s}^{3}$ ) is proportional to $1 / M^{2}$. For a $1 M_{\odot}$ black hole, $\bar{\rho} \sim 10^{16} \mathrm{~g} \mathrm{~cm}^{-3}$, forty times nuclear matter density, whereas for a black hole of $10^{8} M_{\odot}, \bar{\rho} \sim 1 \mathrm{~g} \mathrm{~cm}^{-3}$, the density of water. The conditions required for matter to form a small black hole are much more extreme than for a large one. Three main regimes are being discussed:

(a) Stellar-mass black holes formed after the death of some normal stars.

(b) Super-massive black holes $\left(\sim 10^{6}-10^{10} M_{\odot}\right)$ formed in the centres of galaxies as a result of the processes of galactic dynamics. (Collapse of super-massive stars or relativistic star clusters might also produce high-mass black holes.)

(c) Black holes formed as a result of fluctuations or phase transitions in the early universe when conditions were so extreme that black holes of all masses might have been produced.

We will be concentrating here on the stellar mass black holes of class (a) and the galactic-centre black holes of class (b), since these are the ones most connected with observational searches. However, primordial black holes of class (c) have periodically attracted considerable attention (most recently in connection with gravitational microlensing [22]). For a review of primordial black holes, see the article by Carr [23].

As noted by Blandford in his 1987 review article on astrophysical black holes [24] (to which we refer the reader for coverage of many details which we will not be dealing with here), the discussion of whether or not a particular object is indeed a black hole has a rather different character in the case of the stellar-mass black hole candidates from that for the super-massive ones since, for stellar-mass candidates, the main alternatives to black holes are neutron stars which are rather clearly-specified objects about which much is known (although see our discussion in Section 2), whereas for the super-massive candidates the alternatives (dense star clusters, superstars, "magnetoids", etc.) have less clearly constrained properties. 
In this article, we make no attempt at any complete coverage of the very extensive literature involved; our aim is to give a general outline of the main ideas and lines of investigation in a way particularly aimed at relativists who are not specialist astronomers. The references have been limited to a minimum and are focussed on mentioning main key papers supplemented by a few quite complete but more specific reviews and some recent publications, which do include more extensive references. We apologise in advance to all of those colleagues whose work is not directly referenced.

\section{Stellar-mass black holes}

The study of stellar-mass black hole candidates has been intimately connected with the development of X-ray astronomy and so we will first give a brief history of this.

\subsection{Early history}

Since the Earth's atmosphere is very effective in shielding us from X-rays, it is necessary to put detectors on board space-craft or high-altitude balloons in order to make X-ray observations of astronomical objects. The first such observations were made in 1962 by Giacconi, Gursky, Paolini and Rossi [25] using detectors on board an Aerobee space-rocket and they discovered the existence of discrete sources of X-rays outside the solar system. A succession of subsequent rocket and balloon experiments then confirmed the earlier results and obtained more data. By the end of the 1960s around twenty sources had been identified including Cygnus X-1 which is particularly bright and was found to vary with time.

In 1966 an old 12th-13th magnitude star was identified as the optical counterpart of the brightest X-ray source Scorpio X-1. It was not plausible that the X-rays could be coming from the optical star itself and, in the following year (1967, the year in which pulsars were first discovered), a model was proposed [26] in which they were explained as originating from gas which becomes very hot in the process of being accreted from the observed star onto an undetected binary companion, a compact object which was taken to be a neutron star. To be precise, by "compactness" we mean the ratio of the actual radius of the object to its Schwarzschild radius $r_{s}$ : the smaller this ratio is, the more compact the object is said to be. The term "compact object" is used to refer to white dwarfs, neutron stars and black holes, but we should emphasize that while white dwarfs are much more compact than ordinary stars like the sun, they are not nearly as compact as neutron stars and black holes. Some relative sizes are shown in Fig. 1: neutron stars can come rather close to being black holes.

In 1968, Prendergast and Burbidge [27] argued that the accreted material in this model would be carrying so much orbital angular momentum that it would not fall onto the compact object along radial paths but rather would be drawn into a thin accretion disc around the compact object (see Fig. 2) and then slowly spiral inwards as a result of viscous drag. The viscosity also heats the disc up to temperatures at which it emits 


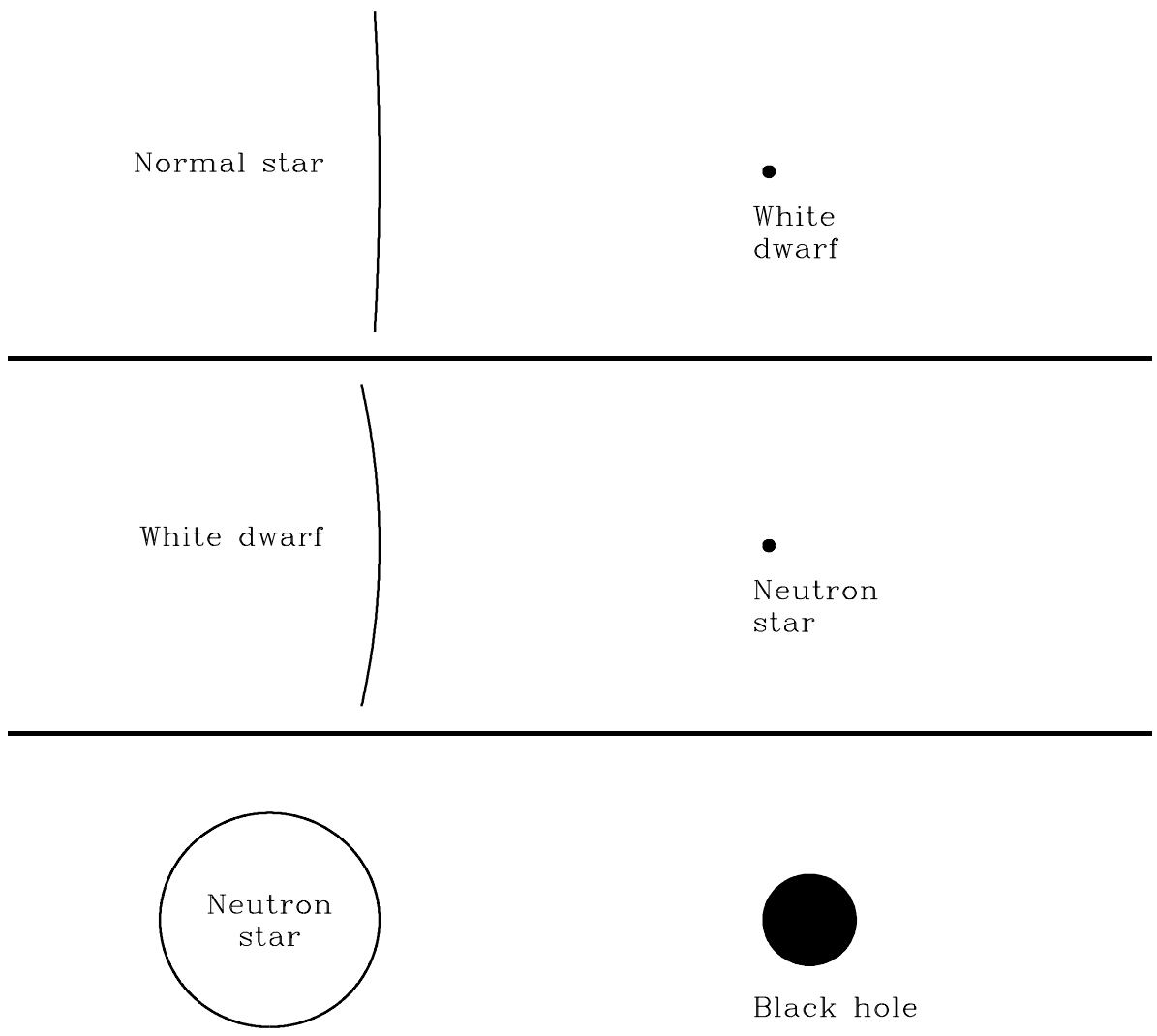

Fig. 1. Relative sizes of normal stars, white dwarfs, neutron stars and black holes having similar masses (we have taken $1.4 M_{\odot}$ ). Note that while white dwarfs are much more compact than normal stars, they are not nearly as compact as neutron stars or black holes which, however, come rather close together.

the observed radiation. (Note that this picture can apply only if the magnetic field of the compact object is not too high; a strong dipole magnetic field would cause the flow to be funnelled down the magnetic axis.) The accretion may be initiated by the optical star swelling up to fill an equipotential surface called the Roche lobe and then losing matter to its companion via the crossing-point in this surface. Gravitational potential energy liberated as the accreted matter spirals down the gravitational potential well of the compact object is converted partly into kinetic energy (mainly rotational) and partly into thermal energy, some of which is then radiated. This can be an extremely efficient energy generation mechanism, particularly if the compact object is a black hole.

However, up to this point, there had not yet been conclusive observational evidence that any observed X-ray sources were, in fact, in close binary systems or were associated with compact objects. The evidence that this was so came from the Uhuru satellite launched in 1970. Uhuru was entirely devoted to X-ray observations and continued to operate for more than three years during which time it found more than three hundred sources and established that many of them were indeed associated with 


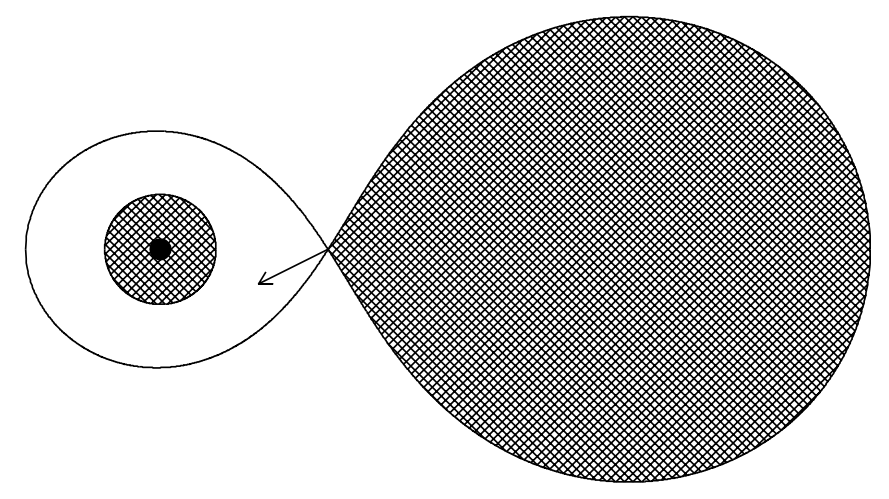

Fig. 2. View down the rotation axis of a binary system in which accretion is taking place. The secondary star has swollen up to fill an equipotential surface called the Roche lobe and is spilling matter across onto the compact object. Because of its high angular momentum, this accreted material forms itself into a rotating disc.

binary systems consisting of an optically-observed star together with an opticallyinvisible companion [28]. Also, short timescale variability was seen in the X-ray emission of many sources which, assuming that variations cannot occur on a timescale shorter than the light crossing time, indicated that they did contain compact objects.

\subsection{Cygnus $X-1$}

During the 1970s and 1980s, particular attention was focussed on the source Cygnus X-1, which appeared to be the strongest candidate for containing a black hole. This source was positively identified with a binary system consisting of a highmass OB supergiant star, HDE 226868, orbiting an unseen companion with an orbital period of 5.6 days. It showed X-ray variability on a range of timescales extending down to one millisecond, indicating that the companion is extremely compact and must be either a neutron star or a black hole [29]. How can one distinguish between these two possibilities? Neutron stars cannot have arbitrarily large mass; there is a maximum above which the pressure can no longer balance gravity. This maximum is currently thought to be somewhere between $1.4 M_{\odot}$ and $2.5 M_{\odot}$ if the neutron star is non-rotating (or rotating only very slowly) and may be raised by up to $25 \%$ if it is rotating rapidly. If one could determine that the mass of a very compact object is above the maximum for a neutron star, then it would presumably have to be a black hole. This is the line of reasoning that was followed with Cygnus X-1 and with various subsequent black hole candidates.

How can the mass of the compact object be established from observations? We know that HDE 226868 is a member of a binary system because its spectrum shows systematic Doppler shifts which are consistent with it moving on a binary orbit under the influence of the unseen companion. From the Doppler shift data, a radial velocity curve can be constructed, giving the variation with time of the component of the star's 
velocity along the line of sight. From this one can extract the orbital period $P$, the semi-amplitude of the curve $K$ and, in principle, the eccentricity of the orbit (although this is extremely small in the case of Cygnus X-1 and, for simplicity, we will neglect it in the following discussion). Next, we make use of Kepler's laws of orbital motion applied to the layout shown schematically in Fig. 3.

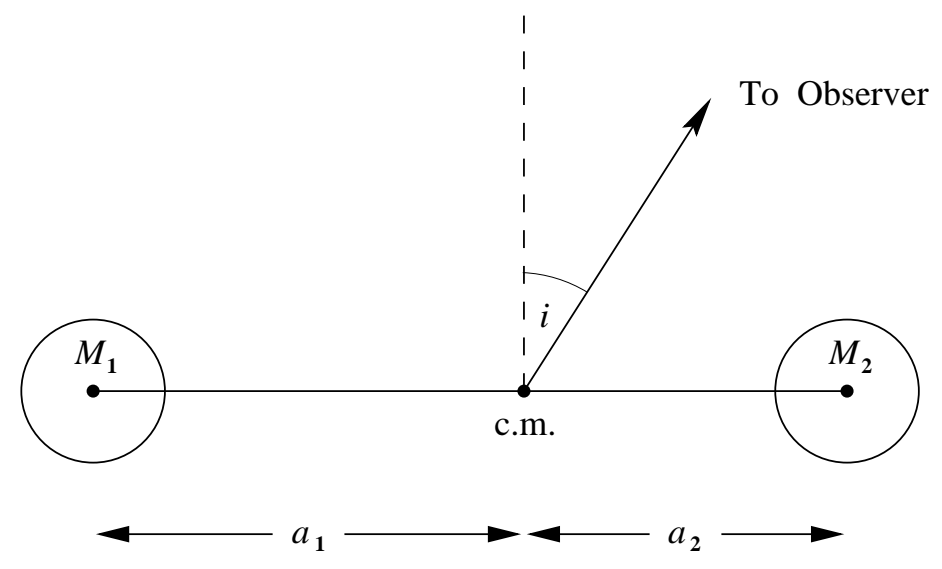

Fig. 3. Schematic picture of the binary parameters as viewed in the orbital plane of the system. The compact object has mass $M_{1}$, the optical star has mass $M_{2}$ and distances are measured from the centre of mass (c.m.) of the system.

Using Kepler's second law, we have that $K=2 \pi a_{2} \sin i / P$ and then, using Kepler's third law, one can obtain a quantity called the mass function:

$$
f\left(M_{1}\right)=\frac{\left(M_{1} \sin i\right)^{3}}{\left(M_{1}+M_{2}\right)^{2}}=\frac{P K^{3}}{2 \pi G} .
$$

For Cygnus X-1, $f\left(M_{1}\right)=(0.252 \pm 0.010) M_{\odot}$. In order to determine the mass of the compact object, it is necessary to supply two further pieces of information: the mass of the optical star and the angle of inclination of the orbit (or other information which allows these to be calculated). Typically, OB supergiants have masses greater than $20 M_{\odot}$ and taking plausible values for the mass of HDE 226868 and for sin $i$ led to the conclusion that the mass of the compact object is probably in the range $9-15 M_{\odot}$, which is well above the maximum for neutron stars quoted earlier. It is very likely that Cygnus X-1 does contain a black hole with a mass of more than $9 M_{\odot}$ but one ought to be cautious and ask what is the minimum possible value of $M_{1}$ consistent with the data.

While OB supergiants do typically have masses greater than $20 M_{\odot}$, it is also possible for them to be considerably less massive without there being any significant change in the spectrum. However, there is another constraint for HDE 226868 which comes from requiring that it should be able to produce the observed luminosity. Its distance from us can be calculated by considering interstellar absorption observed in 
the optical spectrum and this gave around 2.5 kiloparsecs $\left(1\right.$ parsec $\left.\simeq 3 \times 10^{18} \mathrm{~cm}\right)$ with a firm lower limit of about 2 kiloparsecs. One then asks what would be the minimum mass for the star in order that it could produce the observed luminosity if it is 2 kiloparsecs away. According to stellar structure calculations of the time, this came out at $8.5 M_{\odot}$. A lower limit for the mass of the compact object can then be obtained by noting that while the angle of inclination of the orbit is not known, $\sin i$ must certainly be $\leq 1$. This then gives $M_{1} \gtrsim 3.3 M_{\odot}$ which is above the presumed maximum for a non-rotating neutron star making Cygnus X-1 a good candidate for containing a black hole.

In fact, it is possible to do rather better than this if one has observational data of sufficient quality and a detailed analysis of the Cygnus X-1 system was presented in 1986 by Gies and Bolton [30], combining together data from fifty-five observations spread over ten years. By measuring the rotation-broadening of the optical star's absorption spectrum and making certain assumptions about the system, it is possible to derive a value for the mass ratio $q=M_{1} / M_{2}$. Modelling the ellipsoidal modulation of the light curve (resulting from the fact that a non-spherical emitter presents a timevarying effective emitting area to the observer as it goes round its orbit) then allows $\sin i$ to be calculated and hence all of the parameters can be determined consistently with the assumptions made. Proceeding in this way, Gies and Bolton derived a lower limit for $M_{1}$ of $7 M_{\odot}$ with a preferred value of $16 M_{\odot}$.

However, some doubts remain about Cygnus X-1 which make it difficult to determine an accurate mass for the compact object. The optical star is highly evolved and of uncertain mass, does not seem to be filling its Roche lobe and may not be rotating synchronously with the orbital motion; there is no velocity information about the compact object itself. (In the early days there was also a suggestion that there might have been a third object in the system [31].) Now, there is a new generation of rather different candidates for which the determination of accurate masses is easier.

\subsection{Soft $X$-ray transients}

The uncertainties with Cygnus X-1 had mainly been related to the fact that it is in a high-mass X-ray binary (i.e. the "normal" component is a high-mass star). At first sight, low-mass X-ray binaries (LMXBs) would seem to be more straightforward to deal with but they suffer from the major difficulty that the optical light is dominated by that coming from the X-ray irradiated accretion disc which is much brighter than the optical companion, normally making the latter impossible to study. This is not a problem, however, if the source is transient, emitting X-rays for only part of the time. The best current stellar-mass black hole candidates are soft X-ray transients (SXTs), a sub-class of LMXBs which are X-ray luminous for around six months in every $10-50$ years [32]. During quiescence, the accretion disc becomes extremely faint and it is then possible to carry out detailed photometry and spectroscopy of the optical companion. This is still not an easy task, however, because these objects are themselves very faint and it is only recently that the equipment has existed on large 
telescopes to enable such studies to be made. It is widely considered that the current strongest candidate is the source V404 Cygni which is associated with a binary system having a 6.5 day orbital period and has an interval between outbursts of 25 years (or it may be one half of that). The last outburst was in 1989 .

For this sort of system, the assumptions of corotation and of the optical star filling its Roche lobe seem to be rather good and by combining the measurements of rotation-broadening of the absorption spectrum and the ellipsoidal variation of the light curve (best done here at infra-red wavelengths in order to reduce contamination from the accretion disc) it is possible to make a reliable determination of all of the binary system parameters. Proceeding in this way, Shahbaz et al. [33] determined the masses of both components in several SXTs. For V404 Cygni, they measured the mass of the compact object as $12 \pm 2 M_{\odot}$ which is well above the usually accepted mass limit for a neutron star. In the next section, we say something more about this mass limit.

\subsection{The maximum mass for neutron stars}

How much should one trust the upper mass limit for neutron stars? This has been obtained using equations of state for material at very high densities extending above that of nuclear matter where the physics is not very well understood and is poorly constrained by experimental data. Different equations of state give a considerable range of values for the neutron star maximum mass.

In 1974 Rhoades and Ruffini [34] presented an argument designed to circumvent the uncertainty in the equation of state of matter at very high densities and to derive a firm upper mass limit for neutron stars, on the basis only of knowledge which could be considered as really secure. They noted that the equation of state $i s$ thought to be quite well-known at lower densities and so it could be taken as fixed for densities $\rho$ less than some fiducial value $\rho_{0}$, while for $\rho>\rho_{0}$ it was varied so as to obtain the maximum possible mass consistent with the following assumptions:

(i) Gravity is well described by general relativity;

(ii) The equation of state depends on only one parameter (the pressure $p=p(\rho)$ );

(iii) The matter is microscopically stable: $d p / d \rho \geq 0$;

(iv) The equation of state satisfies the causality condition that the speed of sound $c_{s}$ should be less than the speed of light. This was expressed as $c_{s}^{2}=d p / d \rho \leq c^{2}$.

Doing this and taking $\rho_{0}=4.6 \times 10^{14} \mathrm{~g} \mathrm{~cm}^{-3}$, they found the maximum allowed mass to be $M_{\max } \simeq 3.2 M_{\odot}$. For other values of $\rho_{0}$, the result scales roughly as

$$
M_{\text {max }} \simeq 3.2\left(\frac{\rho_{0}}{4.6 \times 10^{14} \mathrm{~g} \mathrm{~cm}^{-3}}\right)^{-1 / 2} M_{\odot} .
$$

A number of questions now arise: 
(a) Is the causality constraint (iv) reasonable? As pointed out by Hartle [35], $(d p / d \rho)^{1 / 2}$ is the phase velocity for small disturbances and is equal to the group velocity (which is the relevant quantity for causality considerations) only if the medium is non-dispersive which is not the case for neutron-star matter. However, even without condition (iv), it is still possible to obtain an upper limit, and this is given by

$$
M_{\max } \simeq 5.3\left(\frac{\rho_{0}}{4.6 \times 10^{14} \mathrm{~g} \mathrm{~cm}^{-3}}\right)^{-1 / 2} M_{\odot}
$$

(b) Do we trust out knowledge of the equation of state up to $4.6 \times 10^{14} \mathrm{~g} \mathrm{~cm}^{-3}$ for bulk high-density matter? A lower threshold figure would be safer. With $\rho_{0}$ referred to $10^{14} \mathrm{~g} \mathrm{~cm}^{-3}$, the basic formula becomes

$$
M_{\max } \simeq 6.8\left(\frac{\rho_{0}}{10^{14} \mathrm{~g} \mathrm{~cm}^{-3}}\right)^{-1 / 2} M_{\odot},
$$

or

$$
\simeq 11.4\left(\frac{\rho_{0}}{10^{14} \mathrm{~g} \mathrm{~cm}^{-3}}\right)^{-1 / 2} M_{\odot},
$$

when combined with (a) above.

(c) Then there is rotation. Friedman and Ipser [36] showed that if one allows for uniform rotation up to the shedding limit

$$
M_{\text {max }} \simeq 8.4\left(\frac{\rho_{0}}{10^{14} \mathrm{~g} \mathrm{~cm}^{-3}}\right)^{-1 / 2} M_{\odot},
$$

with condition (iv) or

$$
\simeq 14\left(\frac{\rho_{0}}{10^{14} \mathrm{~g} \mathrm{~cm}^{-3}}\right)^{-1 / 2} M_{\odot},
$$

without condition (iv).

At this point, it is no longer clear that the situation is safe even for V404 Cygni. Standard realistic neutron star equations of state do, in practice, give masses roughly satisfying the original condition $M_{\max } \lesssim 3.2 M_{\odot}$ even with rotation but now we are back to asking how completely we trust the picture which they represent. Are we really sure that compact stars at these densities do consist of a mixture of neutrons, protons, electrons, mesons, hyperons and other particles held together just by gravity or could another picture be correct? (While it is the strong force, and not gravity, which holds together atomic nuclei, standard neutron star models do not envisage the strong force playing any confining role on a macroscopic scale. In this sense, they are very different from "giant nuclei".) 
A radically different viewpoint was presented by Witten in 1984 [37] with the introduction of the strange star model. Quantum chromodynamics contains the idea of confinement by the strong force which is normally thought of in terms of quarks being confined within nucleons. The idea of the strange star model rests on the hypothesis that strange quark matter (composed of almost equal numbers of up, down and strange quarks) might be the absolute ground state of baryonic matter even at zero pressure. Strange stars would be essentially giant nucleons with quarks being confined inside them but able to move freely in the false vacuum which extends throughout the interior. They could have masses and radii similar to those of standard neutron stars and have been advocated as a viable alternative model for pulsars, although there may be a problem over explaining glitches.

Strange stars are "safe" as far as the mass limit is concerned. Although their equation of state is very different from that for standard neutron star matter, the maximum mass is within the standard range (it is $\sim 2.0 M_{\odot}$ for non-rotating models with some variation depending on uncertain parameter values). However, some effective field theories of the strong force allow for it not only to confine quarks in the normal way but also to confine nucleons (neutrons and protons) at densities well below that of nuclear matter thus giving an equation of state different from the standard one at densities below the values normally taken for $\rho_{0}$. Models based on this idea were introduced in 1989 by Bahcall, Lynn \& Selipsky [38] who named them Q-stars (although note that the "Q" here does not stand for "quark" but for a conserved particle number). These are not safe for the mass limit and might, in principle, have very high masses up to more than $100 M_{\odot}$ as well as being extremely compact with radii down to only 1.4 times those of equivalent black holes [39]. For low mass models, the Q-stars would have an almost constant density throughout but for higher masses the density profile becomes more peaked at the centre. Even if one rules out Q-stars as models for pulsars (they have similar difficulties in this respect as for strange stars) there still remains the possibility that they could be an alternative model for the more massive objects in black-hole-candidate systems.

The significance of the Q-star idea is not so much that it is widely expected that the stellar-mass black hole candidates are really Q-stars (although they might be), but rather that this stands as a concrete counter-example to the normal discussion of the mass limit which it is hard to rule out. It is true that there is a difference between the predicted radii of Q-stars and black holes of similar mass and one might hope to

show that a particular object could not be a Q-star by picking up a signal seeming to come from infalling matter at radii too small to be outside the surface of a Q-star. However, it looks difficult to make this distinction in practice because the difference in radius is so small.

\subsection{Searching for direct evidence of an event horizon}

So far, efforts to demonstrate the existence of stellar-mass black holes have mostly been based on the calculated properties of neutron star models (mainly the mass limit 
but now also the compactness) and this is, at best, an indirect line of reasoning. The real point about black holes is the existence of an event horizon with the properties anticipated from theory and it is this that one would like to demonstrate in a clear way. Calculation of neutron star models depends not only on our physical description of high density matter at regimes which are not well-tested in laboratory experiments but also, crucially, on the use of general relativity to describe strong gravitational fields. If one used Newtonian theory instead of general relativity, then all of the present stellar mass black hole candidates could easily be accounted for as neutron stars even with the most standard equations of state. While we do not believe that Newtonian theory gives correct results under these circumstances, general relativity is not the only possible alternative. Until rather recently, this would have remained a serious uncertainty but now that results from the binary pulsars [40] are placing very serious constraints on alternative theories of gravity, the equation of state represents the largest uncertainty.

What are the prospects for demonstrating the existence of an event horizon in a more direct way? One line of reasoning which has attracted a lot of attention in recent years is the following. What happens to the soft X-ray transients when they are in quiescence? A model has been proposed by Narayan and his colleagues (see [41] for a review) in which the accretion rate is reduced as the SXT enters quiescence and the inner parts of the disc swell up to form a structure which has been called an ADAF (Advection Dominated Accretion Flow) [42]. This flow is very hot but of low density and the energy liberated as material moves down the gravitational potential well is mainly advected inwards with the infalling matter rather than being emitted as radiation. Now, if the compact object is a black hole, this energy simply passes through the event horizon and is lost to view but if it is an object with a solid surface then the infalling matter would be brought to rest at the surface and the excess energy would then be emitted as radiation which could be observed. It is claimed that observations of various sources (V404 Cygni in particular) match very well with the picture of an ADAF around a black hole and that this is strong evidence in favour of the presence of an event horizon. However, this interpretation is still under debate and not everyone is convinced by it.

The coming of X-ray astronomy opened a new window onto the Universe for the study of matter under extreme conditions near to compact objects. As we have seen, it has brought us tantalizingly close to an unequivocal demonstration that stellar mass black holes are really being observed but the final step to being sure beyond reasonable doubt has proved to be very difficult to make. Now, at the end of the twentieth century, we hope and believe that we are on the threshold of opening another new window with the detection of gravitational waves by laser interferometric detectors and also, perhaps, by a new generation of bar detectors [43]. Gravitational waves can tell us directly about dynamical changes in gravitational fields associated with very compact objects and bring us information about the physical nature of the sources which cannot be obtained in any other way. In detail, black holes are supposed to be 
very different from any sort of compact star and the sort of additional information which gravitational wave astronomy could provide may allow us to see these differences more directly.

\section{Super-massive black holes}

Following more than three decades of gradual increase in the number of independent pieces of evidence indicating the existence of super-massive black holes in the centres of some galaxies, there has been a remarkable strengthening of the observational evidence in the last few years which has produced a rather compelling case for the presence of dark massive objects in the nuclei of most - if not all - galactic nuclei. Ironically, black holes have now become perhaps the least exotic candidates for identification with these dark objects.

Such powerful and robust evidence has been obtained chiefly thanks to technological advances in observational techniques and instrumental performance in the different energy bands, leading both to improvements in the accuracy of measurements made with already established observational methods and to the discovery of new - and occasionally unexpected - pieces of information. The improvement of accuracy and sophistication in the modelling and interpretation of data has, of course, been equally crucial.

\subsection{Collecting pieces of evidence}

The suggestion that super-massive black holes are hosted in the nuclei of some galaxies originated at the beginning of the 1960s following the discovery of quasars: the conversion into radiation of the gravitational energy of matter in the potential well of such compact objects appeared to be the most efficient way of producing the huge observed powers from within small volumes.

Several phenomena associated with quasars and, more generally, with powerful sources in the central cores of about $10 \%$ of galaxies - globally referred to as Active Galactic Nuclei (AGNs) - have since contributed to reinforcing the belief that black holes are ultimately responsible for the production of energy and activity in these galactic cores (although alternative and more "conventional" hypotheses have also been proposed).

The main characteristic feature of the AGN phenomenon is the inferred compactness of the sources: luminosities of the order of $10^{46} \mathrm{erg} \mathrm{s}^{-1}$ (more than $10^{12}$ times the luminosity of the sun) are produced from regions less than a light year across $\left(\sim 10^{18} \mathrm{~cm}\right)$. (If the energy is taken to be emitted isotropically, the inferred luminosity goes up to $10^{48} \mathrm{erg} \mathrm{s}^{-1}$.) The most extreme constraint on the compactness comes from the high energy (X-ray) radiation. Up to several per cent of the total power can be emitted in this spectral band with the radiation being highly variable on timescales of less than an hour in some cases, thus setting extremely tight upper limits for the typical dimensions of the region in which the energy is generated. This high 
energy radiation, together with other spectral characteristics, including line emission from gas moving at speeds of thousands of $\mathrm{km} \mathrm{s}^{-1}$, cannot be satisfactorily ascribed to any stellar-related (quasi-thermal) process.

There are two main facts which suggest that the AGN power source is associated with a relativistic potential. Firstly, efficiencies of mass-to-light conversion of the order of $10 \%$ are required in order to satisfy the observational constraints on both the amount of available fuel in the host galaxies and the expected residual masses. These efficiences greatly exceed that of nuclear fusion $(\sim 0.8 \%)$. A black hole, as a single super-massive object, is the most likely cause of the required deep potential well. The tight constraints on the size of the volume involved, the long-term stability of such central masses, the quite coherent structure and lack of periodicities in the observed variability, all argue against it being produced by clustering of "small" bodies. These arguments strongly favour the case for the observed luminosity originating from the extraction of gravitational energy from matter in the potential wells of black holes with masses in the range $\sim 10^{6}-10^{10} \mathrm{M}_{\odot}$ (see [44] and references therein).

Secondly, around $10 \%$ of AGNs appear to be associated with the presence of collimated structures (jets) along which plasma (presumably ejected from the inner nucleus) sometimes moves at relativistic speeds, corresponding to bulk Lorentz factors of the order of 10. The associated bulk kinetic power (and possibly also the magnetic power) can be of the same order as the total luminosities. These structures can extend for up to a million light years (i.e. through about ten orders of magnitude increase in dimension) while remaining reasonably aligned. The jet phenomenon thus requires the presence of a relativistic potential well and a stable preferred axis over long timescales, properties which can consistently be ascribed to the presence of a (spinning?) black hole at the centre of the host galaxy (see [45] and references therein). The jet power might even be extracted directly from the rotational spin energy of the hole itself by means of the Blandford-Znajek mechanism [46].

A further fact supporting the black hole conjecture is that, despite significant phenomenological differences, the fundamental properties of AGNs seem to be remarkably similar over a luminosity range of more than six orders of magnitude (or even greater if one considers the analogy with stellar-mass black hole candidates which we discuss in the Conclusion).

\subsection{Black hole demography}

In recent years, evidence has accumulated for the presence of massive compact objects both in active galaxies (i.e. ones showing signs of activity in the core) and (less predictably and more importantly) in non-active galaxies, for which observations do not reveal any clear sign of non-stellar processes taking place.

The crucial information which provides the clearest signature for not only a central relativistic potential but, indeed, for a super-massive black hole, are measures of (or limits on) the presence of a large, dark and stable mass concentration within a small enough radius, such that the inferred gravitational potential cannot easily be ascribed 
to aggregates (dense clusters) of stars, black holes, brown dwarfs, planets or elementary particles. Constraints on these alternatives come from the requirement that the timescale for cluster evaporation and/or collapse into a single object (depending on the mass, size and density of the clustering objects) should be longer than the age of the system. In at least two galaxies (the Milky Way and NGC 4258) the limits on the size are so tight that the identification of the dark objects with super-massive black holes seems to be almost unavoidable.

The recent indications of the presence of dark massive objects in the centres of galaxies are based on measurements of their effects on the dynamics of stars and/or gas in the inner galactic core, within the comparatively small radius where the potential of the central dark object is dominant $\left(r \sim G M / \sigma^{2} \sim 40 M_{8} \sigma_{100}^{-2}\right.$ parsecs, where $M=10^{8} M_{8} M_{\odot}$ is the mass of the central object and $\sigma=100 \sigma_{100} \mathrm{~km} \mathrm{~s}^{-1}$ is the root mean square orbital speed). Even for the (nearby) Virgo cluster, these observations require an angular resolution better than $\sim 0.2$ seconds of arc. Stars and clouds of gas within this radius have been considered as quasi test particles orbiting a central mass with velocities given by $v^{2}=\alpha G M / r$ where, however, $\alpha$ might need to be determined by detailed modelling for the potential as being due to a point source together with the extended galaxy and with the velocity distribution being anisotropic.

Use of both stellar and gas dynamics provides interesting advantages and simplifications for the modelling and hence for the robustness of the inferred results (see, for example, [47]).

The motion of stars is directly (and almost solely) affected by the potential well of the galaxy and the central mass concentration: stars thus behave basically like point masses in ballistic motion. However, the random-motion component of their speed (only velocity components along the line of sight can be measured) can exceed the bulk-motion one. The velocity field can be significantly anisotropic since encounters between stars are negligible and the relaxation time for the stellar system exceeds the other relevant timescales and, in fact, suitable stellar distributions are known to mimic the presence of a central massive object. Therefore the results obtained from stellar dynamics are significantly dependent on modelling (which, however, is helped by the fact that the point-like mass potential can be considered as stationary, since the growth timescale for the central mass is much longer than the orbital timescale for the stars).

Gas dynamics, on the other hand, can in principle be influenced by forces other than gravity (e.g. radiation pressure, pressure gradients, etc.). However, since internal energy can quite easily be dissipated whereas angular momentum cannot, the gas might plausibly be expected to be relatively cold and in a disc-like structure, with the bulk-motion component dominating the dynamics, thus simplifying difficulties associated with the de-projection of the velocity field. Gas dynamical measures have thus provided, in some cases, less ambiguous evidence for the presence of a central mass and more secure measurements of how much matter is there. 


\subsection{Stellar dynamics}

Thanks to the unprecedentedly high angular resolution and sensitivity of the Hubble Space Telescope (HST), it has been possible to measure the spatial distribution and spectroscopic velocities of stars in the cores of several nearby galaxies. Up to now, $\sim 15$ robust mass determinations have been made plus another $\sim 20$ which are more critically model dependent and these almost invariably point towards the presence of a massive dark object in the centre of the galaxy (i.e. with corresponding mass-to-light ratios greatly exceeding that for the sun), with the inferred masses ranging between $\sim 10^{6}$ and a few $\times 10^{9} \mathrm{M}_{\odot}$ (see [48] and references therein). Among these is the case of the nearby Andromeda galaxy (M31).

Three-dimensional stellar dynamics has also provided the strongest evidence supporting the presence of a (mildly active) black hole in the centre of our own Galaxy (as indeed predicted by Lynden-Bell \& Rees in 1971! [49]). Previous radio and infrared measurements of the gas motion had shown that this was not determined only by the central gravitational potential and thus proved inadequate for reaching a firm conclusion. The new evidence had to be obtained using observations in the near infrared band (performed with the New Technology Telescope at ESO) since our line of sight towards the Galactic Centre is strongly obscured at optical and ultraviolet wavelengths by the presence of dust and gas in the plane of the Galaxy. The motion of individual stars in the central 0.01 parsec of the Galaxy (with speeds exceeding thousands of $\mathrm{km} \mathrm{s}^{-1}$ ) was accurately monitored over a period of a few years, providing measures of the transverse component which, together with spectroscopic radial velocity information, have allowed a remarkably accurate estimate to be made of the central mass: $2.6 \times 10^{6} \mathrm{M}_{\odot}($ Eckart \& Genzel 1998 [50]).

\subsection{Gas discs}

Determinations of the dynamics of gas discs (and a few stellar discs) within the inner $\sim 100$ parsecs of some nearby galaxies have been obtained using HST observations. For several systems, photometric images of (often dusty) discs have been obtained. In some cases, the emission of ionized gas in certain spectral lines is sufficiently bright that unambiguous evidence has been found for Keplerian orbital motion of a thin distribution of gas (quasi-flat and therefore relatively cold) indicating, a posteriori, that the gas dynamics is mainly subject to the gravitational field produced by a central mass.

A remarkable example is the case of M87, in the Virgo Cluster, which shows signs of low-power activity (the most conspicuous being the presence of jets observed in the radio, optical and X-ray bands). Being at such low redshift, a stellar cusp in the giant elliptical host galaxy had long been observed [51] and a central mass of $\sim 10^{9} M_{\odot}$ had been inferred but it was shown that the dynamics could also be accounted for by an anisotropic velocity field. However, the presence of a central mass has now become compelling following the determination of the velocity field of a circumnuclear disc of ionized gas emitting in $\mathrm{H} \alpha$, whose line profiles and corresponding rotation curve are 
extremely consistent with the gas being in Keplerian motion in a plane approximately perpendicular to the jet direction. The measurements made imply the presence of a total mass of $\sim 3.2 \times 10^{9} M_{\odot}$ within the central 3.5 parsecs of the galaxy (Macchetto et al 1997 [52] and references therein).

\subsection{Water maser discs}

An independent observational technique, which has provided one of the strongest cases for the presence of super-massive black holes, is the measurement of gas dynamics by means of the maser-emission line of water at the wavelength of $1.3 \mathrm{~cm}$. Although several maser-emitting discs have now been discovered, the most convincing and robust case remains that of the spiral galaxy NGC 4258, perhaps because its emitting disc is observed edge-on. The nearby spiral galaxy NGC 1068 provides the second best example.

Radio measurements with the VLBA (Very Long Baseline Array) can achieve angular resolutions $\sim 100$ times better than HST (less than half of a milliarcsecond) and show the presence of small $\mathrm{H}_{2} \mathrm{O}$ masers distributed over $\sim 0.1$ parsec, confined within a single (although warped) plane. Also, the VLBA spectral resolution is so accurate that the rotational velocity of the molecular gas can be determined as being Keplerian motion on circular orbits with a precision of better than $1 \%$. The inner edge of the detected disc is at a radial distance of 0.13 parsec. The inferred nuclear mass of $3.6 \times 10^{7} M_{\odot}$ confined within such a small region is inconsistent with being a stable cluster of stars, strongly pointing towards the presence of a black hole (Miyoshi et al. 1995 [53]).

\subsection{X-ray lines}

The measurements described so far are limited to mapping the dynamics of stars and gas at distances larger than $\sim 10^{4}$ times the typical radius of the inferred central black hole. Therefore, while the observations provide some powerful evidence for the presence of massive dark objects even in non-active galaxies, the dynamics of these systems can be well-described in the Newtonian limit and do not provide any direct test for general-relativistic effects.

Most of the phenomenology associated with AGNs only requires the presence of a deep gravitational potential well in which mass-energy can be converted and radiated with high efficiency. Indeed, in Active Nuclei the thermal and ionization properties of gas located at distances larger than light days from the primary luminosity source are those relevant for the emission of radiation at optical and longer wavelengths. However, higher energy emission (typically X-rays) is produced at smaller radii, less than a light hour away from the central engine, where the material is at a higher temperature and the form of the potential well can imprint interesting signatures on the emitted radiation. Measurements with high sensitivity and high energy resolution of the X-ray spectra of nearby AGNs have provided the first direct indication of 
material accreting in a relativistic potential, as well as information about its spatial distribution and accretion properties.

In particular, in the last decade observations have shown the relatively common presence of emission lines in the X-ray band (mostly for nearby Seyfert galaxies) at around 6-7 keV. These features and the shape of the associated broad-band spectrum appear to be fully consistent with a scenario in which a primary X-ray continuum source illuminates the surface of a medium which is optically thick to Thomson scattering and then is partially absorbed, partially Compton reflected and partially re-emitted as line radiation. The strongest line is consistently accounted for as being emission from the $\mathrm{K} \alpha$ fluorescent transition of highly (photo-)ionized iron atoms, which are sufficiently abundant to produce the detected photon flux. The solid angle subtended by the reflecting gas and its relatively low temperature $\left(\lesssim 10^{6} \mathrm{~K}\right)$ also suggest that this can be consistently identified with matter accreting onto a central black hole in the core of an AGN in a probably quasi-Keplerian thin disc.

The profile of a spectral line emitted from the inner parts of the disc can provide information about the gravitational field at the location of the radiating gas (Fabian et al. 1989 [54]). Recent exciting results for such reprocessed spectra followed observations by the ASCA and BeppoSAX X-ray satellites: the sensitivity and spectral resolution provided by the on-board instruments allowed the profile of the iron line to be resolved, convincingly revealing broad and asymmetric (skewed) features with strongly redshifted tails (Tanaka et al 1995 [55]). The line widths imply velocities of the order of a few times $10^{4} \mathrm{~km} \mathrm{~s}^{-1}$, thus suggesting the motion of gas in a strong gravitational potential (alternative hypotheses appear to be unsatisfactory). Furthermore, the line shape can be best accounted for by the predicted effects of Doppler shifts and gravitational redshift. Typical inferred radii for the emitting gas are between a few and about ten times the Schwarzschild radius. In the best studied source, MCG-6-30-15, the red side of the line extends down to such low energies that the inner radius for the emitting gas distribution is set at around $3 r_{s}$. The central mass cannot be directly determined from these measurements; however attempts have been made to infer a black hole spin parameter from data modelling. Although this modelling is probably currently too simplified (more than one plausible scenario has been proposed) and the data are not of high enough quality to derive robust estimates, X-ray spectroscopy does constitute a powerful and promising astrophysical tool to probe the strong gravitational field associated with the nuclei of galaxies and to determine the space-time metric (see [56] and references therein).

\subsection{Some implications}

The phenomenological evidence discussed so far for the existence of super-massive black holes in the centres of galaxies has exciting astrophysical implications and has led to intense theoretical work, both from the point of view of their associated physics ("how do they work?") and of their cosmological role ("how do they form and evolve?"). 
Despite the remaining large uncertainties (mostly related to the data interpretation and lack of statistically complete samples of galaxies) the inferred black hole masses and number density are quantitatively consistent with the requirements imposed by the issue of how they are fuelled and by quasar evolution.

Here, we briefly mention some of the cosmological aspects related to the recent findings which are currently being debated. Several of the most crucial and recently re-stimulated issues are directly related to the inference that there may be large black holes in the nuclei of most (if not all) nearby galaxies. Interestingly, and rather independently, it has been found that about $50 \%$ of nearby galaxies might show some (low level) nuclear activity. Also, the determination of black hole masses has revealed that these are somehow related (with a rather large dispersion and possibly subject to observational selection effects) to the mass of the bulge (or spheroidal) component of the host galaxies, with the inferred black hole masses being a few tenths of a percent of the bulge masses. It appears that the existence and/or the formation of bulges of galaxies is closely related to the central mass concentration in black holes. Questions then arise not only about when and how super-massive black holes were formed, but also (closely connected with this) about how their formation is related to that of the whole host galaxy [57].

Since luminous quasars appear to be present already at cosmological redshift $z \sim 5$, strong requirements are set on the rapidity of the initial black hole formation. Although this might have begun with the collapse or merger of initial seeds of the order of tens of solar masses which then subsequently grew through accretion up to typical masses of $10^{8} M_{\odot}$, probably the most widely-accepted view associates the formation of black holes with the initial collapse of gas possibly left over from the same cloud from which stars initially condensed [58]. Following the initial collapse, the main luminous quasar phase would then take place, with black hole masses growing to $\sim 10^{6}-10^{9} M_{\odot}$. Later activity, corresponding to the most recent AGN phenomena, stimulated for example by mergers and close galaxy encounters, would then constitute a relatively minor event.

\section{Conclusion}

In this article we have described the situation regarding astrophysical evidence for the existence of black holes in two distinct mass ranges corresponding to stellar masses and the masses typical of galactic nuclei. Evidence has been progressively mounting and the case is now rather strong for saying that black holes have indeed been observed, particularly on the super-massive scale where there now seems little alternative to accepting this conclusion in the case of the best candidates. For stellarmass candidates, the case already looks conclusive if one accepts the conventional view of the maximum-mass limit for sufficiently-compact alternatives to black holes. However, the argument leading to this mass limit still leaves some possible scope for doubt. 
While the discussion of the properties of black hole candidates is rather different for the two mass ranges, there are many similarities between the two scales which may be usefully exploited. In particular, great interest has been aroused over the last few years by observations of so-called "micro-quasars" [59] (of which the best current example is the source GRS 1915+105). These are stellar-mass black hole candidate systems which show many of the properties of AGNs (including expulsion of material along the rotation axis) but with characteristically shorter timescales (roughly proportional to the inferred black-hole mass). Because these objects are easier to observe than AGNs (both because they are closer and also because of the shorter timescales involved), this link between the different scales may provide important new insights into how AGNs work.

During the preparation of this article, it was announced that some $\alpha$-process elements (oxygen, magnesium, silicon and sulphur) have been detected in the normal companion star of the binary black-hole candidate Nova Scorpii [60] (thought to be a black hole of mass $5.5-7.9 M_{\odot}[61]$ ). These cannot have originated in the normal star and the most obvious explanation is that they were transferred to it as a result of a supernova explosion of the progenitor of the black hole candidate, thus giving observational confirmation for previous theoretical results suggesting that black holes could result from supernova explosions.

Study of iron-line profiles (as discussed in Section 3) is potentially a powerful diagnostic tool for determining the properties of black hole candidates although it is much easier to observe them for the super-massive objects than for the stellarmass ones where the strength of the iron lines with respect to the continuum is much weaker. X-ray observations with various newly-launched satellites (e.g. Chandra) and future planned missions (XMM, Astro-E but more powerfully Constellation X) will provide a major impetus for this work particularly in connection with AGNs. Other effects connected with black holes which could be fruitful objectives for future observational study (see the review by Rees [58]) include quasi-periodic oscillations of accretion discs around black holes, in both mass ranges, and optical (and possibly X-ray) flashes resulting from the occasional capture and tidal disruption of stars by super-massive black holes. Finally, we hope that gravitational wave astronomy, which is the subject of another article in this volume [43], will soon provide a new way of looking at both stellar-mass candidates (using earth-bound detectors) and supermassive ones (probably needing to wait for detectors in space).

\section{References}

[1] Michell J 1784 Phil. Trans. R. Soc. (London) 7435

[2] Laplace P S 1796 Exposition du Système du Monde (Paris), vol. 2, p. 305

[3] Israel W 1987 in Three Hundred Years of Gravitation eds. S W Hawking, W Israel, Cambridge University Press, p. 199

[4] Schwarzschild K 1916 Preuss. Akad. Wiss. Berlin, Sitzber. 1916189

[5] Einstein A 1915 Preuss. Akad. Wiss. Berlin, Sitzber. 1915844

[6] Lodge O 1921 Phil. Mag. 41549 
[7] Chandrasekhar S 1931 Astrophys. J. 7481

[8] Eddington A S 1935 Observatory 5837

[9] Lemaitre G 1933 Ann. Soc. Sci. (Bruxelles) A53 51

[10] Einstein A 1939 Ann. Math. (Princeton) 40922

[11] Oppenheimer J R and Snyder H 1939 Phys. Rev. 56455

[12] Bergmann P G 1942 An Introduction to the Theory of Relativity Prentice-Hall: New York, pp. 203-4

[13] Kruskal M D 1960 Phys. Rev. 1191743

[14] Harrison B K, Thorne K S, Wakano M and Wheeler J A 1965 Gravitation Theory and Gravitational Collapse University of Chicago Press

[15] Kerr R P 1963 Phys. Rev. Lett. 11237

[16] Israel W 1967 Phys. Rev. 1641776

[17] Carter B 1973 in Black Holes eds. C DeWitt and B.S. DeWitt, Gordon \& Breach, New York, p. 57

Hawking S W 1972 Commun. Math. Phys. 33323

Robinson D C 1975 Phys. Rev. Lett. 34905

[18] Penrose R 1965 Phys. Rev. Lett. 1457

[19] Hawking S W 1966 Proc. R. Soc. A294 511

[20] Geroch R P 1966 Phys. Rev. Lett. 17445

[21] Hawking S W 1974 Nature 24830

[22] Jedamzik K 1998 Phys. Rept. 307155

[23] Carr B J 1993 in The Renaissance of General Relativity and Cosmology eds. G F R Ellis, A Lanza and J C Miller, Cambridge University Press, p. 258

[24] Blandford R D 1987 in Three Hundred Years of Gravitation eds. S W Hawking, W Israel, Cambridge University Press, p. 277

[25] Giacconi R H, Gursky H, Paolini F R and Rossi B B 1962 Phys. Rev. Lett. 9439

[26] Shklovskii I S 1967 Astron. Zhur. 44930

[27] Prendergast K H and Burbidge G R 1968 Astrophys. J. Lett. 151 L83

[28] Bradt H V D and McClintock J E 1983 Ann. Rev. Astron. Astrophys. 2113

[29] Rothschild R E E, Boldt E A, Holt S S and Serlemitsos P J 1974 Astrophys. J. Lett. 189 L13

[30] Gies D R and Bolton C T 1986 Astrophys. J. 304371

[31] Bahcall J N, Dyson F J, Katz J I and Paczynski B 1974 Astrophys. J. Lett. 189 L17

Fabian A C, Pringle J R and Whelan J A J 1974 Nature 247351

[32] Charles P A 1998 in Theory of Black Hole Accretion Disks eds. M A Abramowicz, G Bjornsson and J E Pringle, Cambridge University Press, p. 1

[33] Shahbaz T, Naylor T and Charles P A 1993 MNRAS 265655

Shahbaz T, Naylor T and Charles P A 1994 MNRAS 268756

Shahbaz T, Ringwald F A, Bunn J C, Naylor T, Charles P A and Casares J 1994 MNRAS 271 L10

[34] Rhoades C E and Ruffini R 1974 Phys. Rev. Lett. 32324

[35] Hartle J B 1978 Phys. Repts. 46201

[36] Friedman J L and Ipser J R 1987 Astrophys. J. 314594

[37] Witten E 1984 Phys. Rev. D30 272

Haensel P, Zdunik J L and Schaeffer R 1986 Astron. Astrophys. 160121

Alcock C, Farhi E and Olinto A 1986 Astrophys. J. 310261

[38] Bahcall S, Lynn B W and Selipsky S L 1989 Nucl. Phys. B325 606

Bahcall S, Lynn B W and Selipsky S L 1990 Astrophys. J. 362251

[39] Miller J C, Shahbaz T and Nolan L A 1998 MNRAS 294 L25

[40] Stairs I H, Arzoumanian Z, Camilo F, Lyne A G, Nice D J, Taylor J H, Thorsett S E and Wolszczan A 1998 Astrophys. J. $\mathbf{5 0 5} 352$

[41] Menou K, Quataert E and Narayan R 1999 in Black Holes, Gravitational Radiation, and the Universe eds. B R Iyer and B Bhawal, Kluwer, p. 265

[42] Narayan R and Yi I 1994 Astrophys. J. 428 L13

Abramowicz M A, Chen X, Kato S, Lasota J-P and Regev O 1995 Astrophys. J. 438 L37

[43] Schutz B F 1999 Class. Quantum Grav. (this volume)

[44] Rees M J 1984 Ann Rev Astron Astrophys 22471 
Blandford R D 1990 Active Galactic Nuclei - 20 th SAAS-FEE Course eds. T J-L Courvoisier and M Mayor, Springer-Verlag

Krolik J H 1999 Active Galactic Nuclei: From the Central Black Hole to the Galactic Environment Princeton Series in Astrophysics, Princeton University Press

[45] Begelman M C, Blandford R D and Rees M J 1984 Rev. Mod. Phys. 56255

[46] Blandford R D and Znajek R L 1977 MNRAS 179433

[47] Kormendy J and Richstone D O 1995 Ann. Rev. Astron. Astrophys. 33581

[48] Richstone D O et al 1998 Nature 39514

van der Marel R 1998 Proc. IAU Symp. 186: Galaxy Interactions and High and Low redshifts eds B Sanders and J Barnes, Kluwer, in press

[49] Lynden-Bell D and Rees M J 1971 MNRAS 152461

[50] Eckart A and Genzel R 1998 The Central Parsecs eds. H Falcke, A Cotera, W Huschl, F Melia and M Rieke, ASP Conference Series, in press

[51] Sargent W L W, Young P J, Lynds C R, Boksenberg A, Shortridge K and Hartwick F 1978 Astrophys. J. 221731

[52] Macchetto F, Marconi A, Axon D J, Capetti A, Sparks W and Crane P 1997 Astrophys. J. 489 579

[53] Miyoshi M, Moran J, Herrnstein J, Greenhill L, Nakai N, Diamond P and Inoue M 1995 Nature 373127

[54] Fabian A C, Rees M J, Stella L and White N E 1989 MNRAS 238729

[55] Tanaka Y et al 1995 Nature $\mathbf{3 7 5} 659$

[56] Fabian A C 1998 Eighth Astrophysical Conference on Accretion Processes in Astrophysical Systems: Some Like It Hot eds. S Holt and T R Kallman, AIP Conf. Proc. 431, 246

[57] Blandford R D 1999 to appear in ASP Conference Series eds. D Meritt, M Valluri and J Sellwood astro-ph/9906025

Salucci P, Szuszkiewicz E, Monaco P and Danese L 1999 MNRAS 307637

[58] Rees M J 1999 to appear in Black Holes and Relativity - Proceedings of Chandrasekhar Memorial Conference, Chicago, Dec. 1996 ed. R M Wald [astro-ph/9701161]

[59] Mirabel I F and Rodriguez L F 1999 Ann. Rev. Astron. Astrophys 37

[60] Israelian G, Rebolo R, Basri G, Casares J and Martin E L 1999 Nature 401142

[61] Shahbaz T, van der Hooft F, Casares J, Charles P A and van Paradijs J 1999 MNRAS 30689

\section{Acknowledgments}

We gratefully acknowledge many helpful discussions with colleagues in Oxford, Cambridge, Trieste and elsewhere. Financial support for our work is provided by the Italian Ministero dell'Università e della Ricerca Scientifica e Tecnologica. 